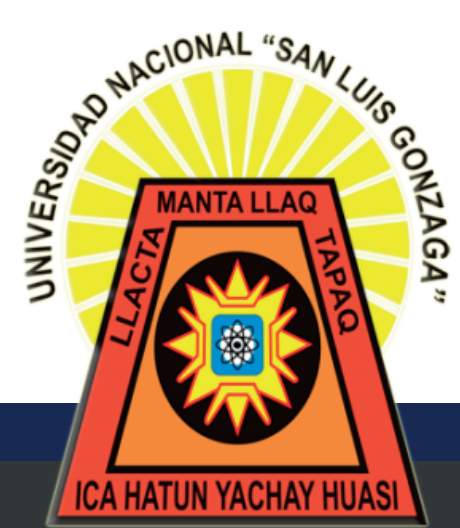

p-ISSN 2223-2893

e-ISSN 2225-6989
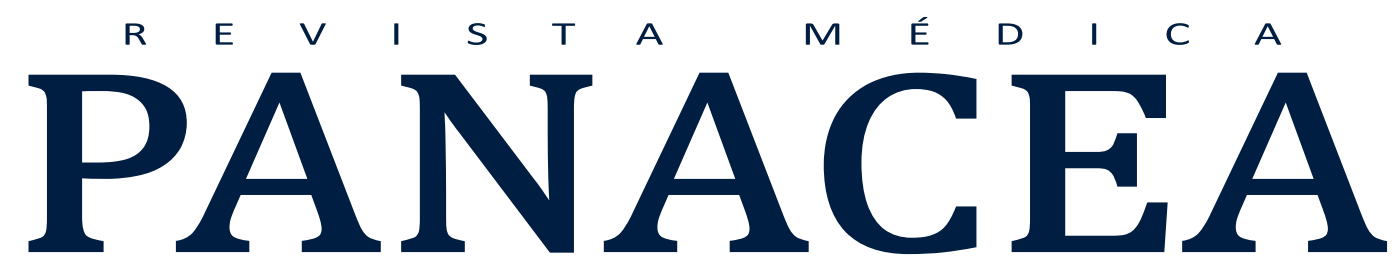

UNIVERSIDAD NACIONAL SAN LUIS GONZAGA. ICA, PERÚ

CARTA AL EDITOR:

\title{
LOS HALLAZGOS PATOLÓGICOS ORALES Y EXTRAORALES MEDIANTE LAS RADIOGRAFÍAS PANORÁMICAS PREVIENEN COMPLICACIONES BUCOMAXILOFACIALES Y ACCIDENTES CEREBROVASCULARES.
}

\section{VOLUMEN 10 NÚMERO 1 PUBLICACION CUATRIMESTRAL ENERO - ABRIL 2021}

\section{AUTORES:}

EDGAR MARTÍN HERNÁNDEZ HUARIPAUCAR

BLADIMIR DOMINGO BECERRA CANALES

NÉSTOR OLIVER GONZALES AEDO

INDEXADA EN:
latindex
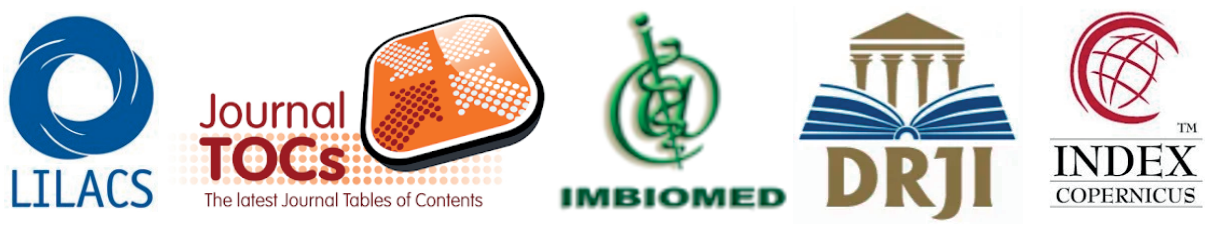
Google

REVISTAS.UNICA.EDU.PE 


\title{
CC Este trabajo es licenciado bajo Licencia Creative Commons Atribución - No Comercial - 4.0 \\ LOS HALLAZGOS PATOLÓGICOS ORALES Y EXTRAORALES MEDIANTE LAS RADIOGRAFÍAS PANORÁMICAS PREVIENEN COMPLICACIONES BUCOMAXILOFACIALES Y ACCIDENTES CEREBROVASCULARES.
}

\author{
Hernández-Huaripaucar Edgar Martín ${ }^{1, a, b}$, Becerra-Canales Bladimir Domingo 1,a,b, Gonzales-Aedo Néstor Oliver ${ }^{1, c}$. \\ 1. Facultad de Odontología, Universidad Nacional "San Luis b. Investigador RENACYT \\ Gonzaga" de Ica-Perú. \\ a. Doctor en Salud Pública \\ c. Magíster en Odontología
}

DOI: 10.35563/rmp.v10i1.409

\section{Correspondencia: \\ Edgar Hernández \\ Huaripaucar \\ Angostura I Etapa K'-3, \\ Subtanjalla, Ica-Perú. \\ Telefono: 956017238. \\ Correo Electrónico: \\ edgar.hernandez@unica.ed}

Contribuciones de autoría: HHEM, BCBD y GANO: participó en todo el proceso de la elaboración de la carta al editor, desde la concepción de la idea hasta el envío a la revista.

Conflicto de intereses: no existen conflictos de intereses del autor o autores de orden económico, institucional, laboral o personal.

Financiamiento:

Autofinanciado.

\section{Cómo citar:}

Hernández-Huaripaucar E M, Becerra-Canales B D, Gonzales-Aedo N O. Los hallazgos patológicos orales y extraorales mediante las radiografías panorámicas previenen complicaciones bucomaxilofaciales

a $c$ i d e n t cerebrovasculares. Rev méd panacea. 2020;10(1) 62-63. DOI:

10.35563/rmp.v10i1.409

\section{Sr. Editor:}

La presente comunicación tiene como motivación dar a conocer las implicancias benéficas de un adecuado diagnostico imagenológico odontológico usando radiografías panorámicas, que contribuye desde la prevención de patologías orales hasta de accidentes cerebrovasculares (ACV), a partir de una atención odontológica rutinaria. Al odontólogo u estomatólogo la radiografía panorámica según Rushton (1) le permite "ejecutar estudios epidemiológicos radiográficos para determinar la prevalencia de anomalías dentarias, óseas u otros tipos de patologías maxilofaciales en niños, adultos y ancianos", lo cual es valioso para conocer las condiciones bucales de diversas poblaciones, permitiendo estudiar, diagnosticar y tratar precoz y adecuadamente algunas patologías bucomaxilofaciales, evitando que lleguen a cirugías más complicadas y secuelas mayores.

El uso habitual de la radiografía panorámica permite descubrir alteraciones y patologías dentales inadvertidas o asintomáticas en pacientes que acuden rutinariamente a una atención odontológica, permitiendo al profesional clínico la observación de estructuras anatómicas anormales o patológicas que no son posibles detectarlos clínicamente a simple vista. Esto mejora el diagnóstico y plan de tratamiento, favorece el diagnóstico y tratamiento preventivo de algunas patologías bucomaxilofaciales y evita cirugías de mayor riesgo y gravedad, así como secuelas y complicaciones irreversibles.

Este beneficio preventivo que nos permite el uso rutinario de las radiografías panorámicas se extiende también a nivel extraoral, pues el cirujano dentista durante una exploración radiológica rutinaria en un paciente odontológico, mediante las radiografías panorámicas, puede detectar la formación de placas de ateromas depositadas en la bifurcación de la arteria carótida, pudiendo tener la posibilidad de derivarlo al cardiólogo oportunamente y contribuir en la prevención de un eminente ACV que pudiera implicar un grave riesgo de morbilidad y mortalidad cerebrovascular para el paciente $(2,3,4)$.

En el actual contexto de la pandemia por la COVID-19, donde cada día se sabe un poco más de esta enfermedad, en un Manual COVID-19 para el neurólogo general, la Sociedad Española de Neurología (SEN) reportó que los ACV isquémicos constituyen la segunda manifestación neurológica más frecuente en pacientes con COVID-19 (5),(6); por lo que existe una razón más para prevenir los ACV en toda la población mundial con riesgo de infección por coronavirus (SARS-CoV-2).

Por la relevante utilidad preventiva de las radiografías panorámicas sobre los ACV debería convertirse en una práctica rutinaria protocolizada en el proceso de diagnóstico oral y extraoral en todas las atenciones odontológicas de los servicios públicos y privados; no obstante, esta propuesta refleja la opinión personal de los autores, pero avalada por muchos otros investigadores a nivel mundial, que consideran que las radiografías panorámicas son herramientas valiosas para el estudio de la estructura ósea y dental del macizo maxilofacial y de la región cervical retromandibular $(7,8,9)$.

Se concluye que se debe fomentar y valorar más el uso rutinario y protocolizado de las radiografías panorámicas en las atenciones odontológicas porque sus eventuales hallazgos en boca y cuello podrían prevenir patologías mayores y salvar la vida de los pacientes. Esta comunicación representa un trabajo original e inédito que no ha sido publicado, ni enviado a otra revista y carece de conflictos de interés. 


\section{REFERENCIAS BIBLIOGRÁFICAS}

1. Rushton V, Horner K, Worthinton H V. Screening panoramic radiology of adults in general dental practice radiological findings. British dental Journal. 2001 May; 190 (9): 495-501.

2. Bravo-Valenzuela $\mathrm{M}$, Reyes-Velásquez J, Valladares-Pérez $\mathrm{S}$. Radiografía panorámica para la detección de ateroma calcificado carotídeo bilateral: reporte de un caso inusual. Revista de Investigación Oral. 2020 noviembre - diciembre; 9 (6): 511-15 [citado 2021 abril 21]; Disponible en: http://search.ebscohost.com/login.aspx?direct=true $\& \mathrm{db}=\mathrm{e}$ dsbas\&AN =edsbas.59751C69\&site=eds-live

Leandro MA. Prevalencia de ateromas en radiografías panorámicas en pacientes mayores de 40 años que acuden a

3. un Centro Radiológico Privado, Huánuco 2017. [Tesis de titulación]. Huánuco: Universidad de Huánuco; 2018. 91 p. [citado 2021 abril 21]. Disponible en: http://repositorio.udh.edu.pe/123456789/1312

4. Zúñiga J I. Prevalencia de placas de ateroma calcificadas en la arteria carótida, en radiografías panorámicas de pacientes mayores de 40 años de edad, en el centro radiológico "Innova Radiografía Dental 3D" Cuenca-Ecuador, en el período 2013-2015. [Trabajo de Titulación - Odontología]. Cuenca: Universidad Católica de Cuenca; 2016. 107 p. [citado 2021 abril 21]. Disponible en: https://dspace.ucacue.edu.ec/handle/ucacue/4851

5. García-Moncó J, Erro M, García D, Ezpeleta D. Cuadros clínicos neurológicos asociados a la infección por SARS-CoV-2. In: Ezpeleta D, editor. Manual COVID-19 para el neurólogo general. Madrid: Ediciones SEN; 2020. p. 36-46.

6. Bravo-Valenzuela $\mathrm{M}$, Reyes-Velásquez J, Valladares-Pérez $\mathrm{S}$. Panoramic radiography for detection of bilateral carotid calcified atheroma: An unusual Case Report. Journal of Oral Research. 2020; 9(6): 511-15.

7. Fuentes $R$, Arias $A$, Borie-Echevarría E. Radiografía Panorámica: Una herramienta invaluable para el estudio del componente óseo y dental del territorio maxilofacial. International Journal of Morphology. 2021; 39(1): 268-73.

8. Garbelotti-Junior S, Ribeiro-Moreira W, Aguiar H, Olave E, Queiroz D. Prevalencia de lesiones ateroscleróticas carotídeas en radiografía panorámica y su relación con factores de riesgo cardiovascular en brasileños. International Journal of Morphology. 2020; 38(6):1560-1565.

9. Llanos Vera, C. E. Prevalencia de anomalías dentales evaluadas en radiografías panorámicas según su forma y número en pacientes atendidos en el Centro de Formación Odontológica Universidad Católica Santo Toribio de Mogrovejo, 2016-2017. [Tesis de segunda especialidad profesional en Ortodoncia y Ortopedia Maxilar]. Chiclayo: Universidad Católica Santo Toribio de Mogrovejo, 2020. 43 p. [citado 2021 abril 21]. Disponible en: http://hdl.handle.net/20.500.12423/2668 